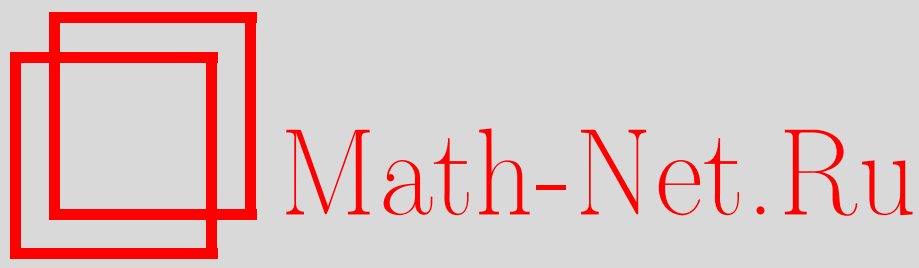

А. М. Ильин, Б. И. Сулейманов, Зарождение контрастных структур типа ступеньки, связанное с катастрофой сборки, Матем. сб., 2004, том 195, номер 12, 27-46

DOI: https://doi.org/10.4213/sm863

Использование Общероссийского математического портала Math-Net.Ru подразумевает, что вы прочитали и согласны с пользовательским соглашением http://www . mathnet.ru/rus/agreement

Параметры загрузки:

IP : 54.198 .64 .247

26 апреля 2023 г., 18:08:17 
УДК 517.928

\author{
А.М. Ильин, Б.И. Сулейманов
}

\title{
Зарождение контрастных структур типа ступеньки, связанное с катастрофой сборки
}

\begin{abstract}
Рассматривается специальное решение обьпновенного диффференциального уравнения $u_{x x}^{\prime \prime}=u^{3}-t u-x$, которое связано с решениями шшрокого ряда дифференциальных уравнений с частньми производньпи с малым параметром. Функция $u(x, t)$ является главным членом асимптотики по малому параметру этих решений около точек сборки предельного решения. Доказывается существование этой специалной функции $u(x, t)$. Строится и обосновьвается ее равномерная асимптотика на бесконечности.

Библиография: 19 названий.
\end{abstract}

\section{Введение}

В статье исследовано специальное решение обцкновенного дифференциального уравнения (ОДУ)

$$
u_{x x}^{\prime \prime}=u^{3}-t u-x .
$$

Это определенное на всей плоскости $(x, t)$ решение ОДУ $(0.1)$ связано с решениями довольно широкого ряда уравнений математической физики [1] с малым параметром. Оно является главным членом внутренних асимптотических разложений в случае, когда их внешние разложения имеют особенности типа сборки [2]. К числу таких уравнений относится, в частности, уравнение

$$
\varepsilon^{2}\left(\frac{\partial^{2} U}{\partial x_{1}^{2}}+\frac{\partial^{2} U}{\partial x_{2}^{2}}\right)+f(X, U)=0, \quad X=\left(x_{1}, x_{2}\right), \quad \varepsilon \ll 1 .
$$

На примере эвристического анализа решений этого уравнения поясним значение и особенности поведения исследуемого решения ОДУ (0.1).

Уравнение (0.2) имеет асимптотическое решение вида

$$
U=u_{0}(X)+\sum_{j=1}^{\infty} u_{j}(X) \varepsilon^{j}
$$

где $u_{0}(X)$ есть корень уравнения

$$
f\left(X, u_{0}\right)=0
$$

Работа вьполнена при поддержке Российского фонда фундаментальных исследований (гранты № № 02-01-00768, 03-01-00716) и Программы поддержки ведущих научных школ Р $\Phi$ (грант № НШ-1446.2003.1). 
Наиболее просто обстоит дело, когда уравнение (0.4) имеет единственный корень, которьй удовлетворяет условию устойчивости

$$
f_{U}\left(X, u_{0}(X)\right)<0 .
$$

(Например, в [3] показано, что в этом случае при дополнительных ограничениях весьма обшего вида решение задачи Дирихле для уравнения (0.2) везде, кроме узкого пограничного слоя, выходит на режим, описьваемьй асимптотическим разложением (0.3).)

Другой типичной [2] ситуацией является наличие точки сборки, из которой на плоскости $X$ исходят две кривые, разделяющие окрестность такой точки на две части: внутри одной решение уравнения (0.4) по-прежнему имеет единственный устойчивый корень $u_{0,0}(X)$, а внутри другой - три корня

$$
u_{0,1}(X)<u_{0,2}(X)<u_{0,3}(X) .
$$

Наименьший и наибольший из них являются гладкими продолжениями корня $u_{0,0}(X)$ через одну из границ области однозначности решения уравнения $(0.4)$. Тогда для построения полной асимптотики решения уравнения (0.2) необходимо исследование решения вблизи такой точки сборки (ср. с работами [4]-[9], где были рассмотрены схожие ситуации). $\mathrm{K}$ примеру, пусть точка сборки - это начало координат, $u_{0}(0,0)=0$, а ряд Тейлора функции $f\left(x_{1}, x_{2}, U\right)$ в этой точке имеет вид

$$
f\left(x_{1}, x_{2}, U\right)=x_{1}+x_{2} U-U^{3}+\cdots,
$$

что с точностью до линейных преобразований соответствует ситуации общего положения.

В естественных внутренних переменных

$$
x=\frac{x_{1}}{\varepsilon^{3 / 4}}, \quad t=\frac{x_{2}}{\varepsilon^{1 / 2}}, \quad v=\frac{u}{\varepsilon^{1 / 4}}
$$

уравнение для главного члена асимптотики - это как раз ОДУ (0.1).

Вне малой окрестности начала координат в области трехзначности корней уравнения (0.4) возникает проблема, касаюшаяся внешшего разложения (0.3): для его однозначного определения внутри этой области необходимо указать линию

$$
x_{1}=\varphi\left(x_{2}\right),
$$

по разные стороны от которой главными членами внешнего разложения $(0.3)$ будут две разные устойчивые ветви решения уравнения $(0.4)$ - корни $u_{0,1}(X)$ и $u_{0,3}(X)$. (Таким образом, внешнее разложение $(0.3)$ определяется гладким вне критической кривой (0.5) корнем уравнения (0.4).) Но проблема нахождения функции $\varphi$ легко решается с помощью простого соображения, очень похожего на то, что используется для определения фронта ударной волны в газодинамических задачах с малой вязкостью [10]-[12].

Для этого надо задаться вопросом о гладком в окрестности кривой (0.5) асимптотическом разложении решения (0.2), сшиваюшем разрывное внешнее разложение $(0.3)$ (пусть для определенности при $x_{1}>\varphi\left(x_{2}\right)$ главный член разложения $(0.3)$ есть корень $\left.u_{0,3}(X)\right)$. 
Растянутой переменной для такого сшиваюшего разложения является, очевидно, переменная

$$
y=\frac{x_{1}-\varphi\left(x_{2}\right)}{\varepsilon},
$$

а его главным членом - гладкое при всех $y$ решение $w\left(x_{2}, y\right)$ ОДУ

$$
\left(1+\varphi^{\prime}\left(x_{2}\right)^{2}\right) w_{y y}+f\left(\varphi\left(x_{2}\right), x_{2}, w\right)=0,
$$

удовлетворяющее условиям

$$
\begin{gathered}
\lim _{y \rightarrow-\infty} w\left(x_{2}, y\right)=u_{0,1}\left(\varphi\left(x_{2}\right), x_{2}\right), \\
\lim _{y \rightarrow \infty} w\left(x_{2}, y\right)=u_{0,3}\left(\varphi\left(x_{2}\right), x_{2}\right), \\
\lim _{y \rightarrow \pm \infty} w_{y}^{\prime}\left(x_{2}, y\right)=0 .
\end{gathered}
$$

После умножения обеих частей ОДУ $(0.6)$ на $w_{y}$ и интегрирования полученного уравнения на промежутке от $-\infty$ до $\infty$, учитьвая условия $(0.7)-(0.9)$, получаем, что критическую кривую (0.5) надо определять из известного правила равных плошадей

$$
\int_{u_{0,1}\left(\varphi\left(x_{2}\right), x_{2}\right)}^{u_{0,3}\left(\varphi\left(x_{2}\right), x_{2}\right)} f\left(x_{2}, \varphi\left(x_{2}\right), w\right) d w=0
$$

Этот вывод о выборе функции (0.5) косвенно подтверждают также результаты работ [13]-[15], объектом исследования которых являлись решения краевых задач для уравнения (0.2) в областях, где решения (0.3) многозначны: по разные стороны от кривой, определяемой именно по правилу (0.10), внешние асимптотические разложения этих решений задаются двумя разными рядами (0.3). В последние годы подобные - вида сглаженных ударных волн - асимптотические решения уравнений с малым параметромпри производных часто назьвают контрастнылми структурами типа ступеньки.

Изучаемое в настояшей статье специальное решение ОДУ (0.1) описывает зарождение такого сорта контрастных структур, связанное с катастрофой сборки. Из обшей идеологии метода согласования [8] следует, что поведение этого решения ОДУ $(0.1)$ при $x^{2}+t^{2} \rightarrow \infty$ должно во многом походить на поведение описанного вьше внешнего разложения асимптотического решения уравнения (0.2).

Например, понятно, что главным членом асимптотики данной функции $u(x, t)$ при $x^{2}+t^{2} \rightarrow \infty$ всюду, кроме окрестности одной линии, является гладкий корень $H(x, t)$ кубического уравнения

$$
H^{3}-t H-x=0 .
$$

Сама же эта линия, расположенная внутри области

$$
-\frac{2 t^{3 / 2}}{\sqrt{27}} \leqslant x \leqslant \frac{2 t^{3 / 2}}{\sqrt{27}}, \quad t>0,
$$

трехзначности решения (0.11), также может быть определена с помощью правила равных плошадей. Действительно, переход при $t \gg 1$ к автомодельным переменHЫIM

$$
s=\frac{x}{t^{3 / 2}}, \quad u=t^{1 / 2} v, \quad H=t^{1 / 2} f
$$


сводит уравнение (0.11) к кубическому уравнению

$$
f^{3}-f-s=0
$$

а ОДУ (0.1) - к сингулярно возмушенному ОДУ

$$
t^{-4} v_{s s}=v^{3}-v-s
$$

Из вида правой части ОДУ (0.14) ясно, что критическая кривая будет иметь вид $x=s_{p} t^{3 / 2}$ и что константа $s_{p}$ находится из уравнения

$$
\frac{f_{1}^{4}\left(s_{p}\right)}{4}-\frac{f_{1}^{2}\left(s_{p}\right)}{2}-s_{p} f_{1}\left(s_{p}\right)=\frac{f_{3}^{4}\left(s_{p}\right)}{4}-\frac{f_{3}^{2}\left(s_{p}\right)}{2}-s_{p} f_{3}\left(s_{p}\right),
$$

где $f_{1}(s)$ и $f_{3}(s)$ суть минимальньй и максимальный из трех корней уравнения $(0.13)$ соответственно. (Правило равных плошадей (0.15) из уравнения (0.13) выводится так же, как выше правило (0.10) было выведено из уравнения (0.2).)

Из уравнений $(0.13),(0.15)$ и из условия расположения критической кривой внутри области трехзначности решения уравнения (0.13) следует, что

$$
s_{p}=f_{2}\left(s_{p}\right)=0
$$

Таким образом, выходит, что критической линией должен быть луч

$$
L:(x=0, t>0) \text {. }
$$

Корень $H(x, t)$ уравнения $(0.13)$, всюду вне луча $L$ являющийся главным членом асимптотики при $x^{2}+t^{2} \rightarrow \infty$ интересующего нас решения (0.1), будет, очевидно, нечетной функцией переменной $x$. С учетом вида уравнения $(0.1)$ логично поэтому ожидать, что нечетным по $x$ должно быть и само это решение уравнения $(0.1)$. Данньй корень $H(x, t)$ монотонно растет по переменной $x$. И продолжая аналогии с газодинамическими задачами с малой вязкостью, решения которых в окрестности фронта ударной волны монотонны [12; 112$]$, следует ожидать, что нужное нам решение ОДУ (0.1) также будет монотонно растущей по переменной $x$ функцией.

Для каждого фиксированного значения $t$ теорема существования монотонно возрастающего и нечетного по переменной $x$ решения ОДУ $(0.1)$ была доказана в [16]. Здесь, в $\S 2$, приводится несколько упрошенное доказательство этой теоремы, но главным содержанием статьи является исследование равномерной и полной асимптотики данного специального решения ОДУ (0.1) при $x^{2}+t^{2} \rightarrow \infty$.

Для того чтобы избежать повторений, в дальнейшем, за исключением особо оговоренных случаев, все рассматриваемые функции будем считать бесконечно дифференцируемыми, постоянные - независимыми как от $x$, так и от $t$, а под монотонностью и нечетностью будем иметь в виду монотонность и нечетность по переменной $x$. 


\section{§1. Вспомогательные леммы}

Лемма 1.1. Пусть при $x \geqslant x_{1}>-\infty$ функиия и(x) удовлетворяет неравенству

$$
u^{\prime \prime}(x) \geqslant \beta^{2} u(x)
$$

для некоторого $\beta>0$ и при $x \rightarrow \infty$

$$
u(x)=o(\exp (\beta x))
$$

Тогда при $x \geqslant x_{1}$ справедлива оченка

$$
u(x) \leqslant\left|u\left(x_{1}\right)\right| \exp \left(\beta\left(x_{1}-x\right)\right)
$$

ДокАЗАТЕЛЬство. Прежде всего отметим, что не существует точки, в которой функция $u(x)$ положительна, а ее производная неотрицательна. Допустим противное: пусть $u\left(x_{2}\right)>0, u^{\prime}\left(x_{2}\right) \geqslant 0$ для некоторой точки $x_{2} \geqslant x_{1}$. Тогда в силу неравенства (1.1) функция $u(x)$ и ее производная монотонно растут при $x \geqslant x_{2}$ и поэтому положительны. Пусть $x_{3}>x_{2}$ - некоторая точка. Ясно, что существует положительная постоянная $\gamma>0$ такая, что $u\left(x_{3}\right)>\gamma, u^{\prime}\left(x_{3}\right)>\beta \gamma$. Обозначим $v(x)=u(x)-\gamma \exp \beta\left(x-x_{3}\right)$. Тогда $v\left(x_{3}\right)>0, v^{\prime}\left(x_{3}\right)>0$, а из неравенства (1.1) следует, что $v^{\prime \prime}(x)>\beta^{2} v(x)$. Поэтому $v(x)>0$ при $x>x_{3}$, что противоречит условию (1.2).

Если $u\left(x_{1}\right) \leqslant 0$, то $u(x) \leqslant 0$ всюду при $x \geqslant x_{1}$. Действительно, в противном случае сушествовала бы точка $x_{2}>x_{1}$ такая, что $u\left(x_{2}\right)>0, u^{\prime}\left(x_{2}\right) \geqslant 0$. Как показано вьше, это невозможно. Итак, в этом случае $u(x) \leqslant 0$ и, очевидно, неравенство (1.3) выполнено.

Остается рассмотреть единственную оставшуюся возможность, когда $u\left(x_{1}\right)>0$, $u^{\prime}\left(x_{1}\right)<0$. В силу изложенного вьше производная $u^{\prime}(x)$ остается отрицательной до тех пор, пока функция остается положительной. Возможны лишш два варианта:

1 ) либо функция $u(x)$ где-нибудь обрашается в нуль;

2) либо $u(x)>0$ всюду при $x \geqslant x_{2}$.

1) Если функция $u(x)$ в какой-нибудь точке обратится в нуль, то пусть $x_{2}-$ такая точка, что $u\left(x_{2}\right)=0$, а $u(x)>0$ при $x_{1} \leqslant x<x_{2}$. Так же, как показано выше (с заменой $x_{1}$ на $x_{2}$ ), следует заключить, что $u(x) \leqslant 0$ всюду при $x \geqslant x_{2}$, так что при $x \geqslant x_{2}$ неравенство (1.3) выполнено.

Для того чтобы проверить неравенство (1.3) при $x_{1} \leqslant x \leqslant x_{2}$, умножим неравенство (1.1) на $u^{\prime}(x)$ и проинтегрируем результат от $x<x_{2}$ до $x_{2}$. Получаем неравенство

$$
u^{\prime}(x)^{2} \geqslant \beta^{2} u^{2}(x),
$$

откуда следует, что $u^{\prime}(x)+\beta u(x) \leqslant 0$, или, что эквивалентно, $(\exp (\beta x) u(x))_{x}^{\prime} \leqslant 0$. Интегрируя последнее неравенство от $x_{1}$ до $x$, получаем оценку (1.3).

2 ) В этом случае $u(x)>0, u^{\prime}(x)<0$ всюду при $x \geqslant x_{1}$. Следовательно, положительная функция $u(x)$ не возрастает и в силу неравенства (1.1) ее предел при $x \rightarrow \infty$ равен нулю. (Иначе этот предел равен $\mu>0$, но тогда $u^{\prime \prime}(x) \geqslant$ $\beta^{2} \mu>0$, что противоречит убыванию $u(x)$.) 
Умножая неравенство (1.1) на $u^{\prime}(x)$ и интегрируя результат от $x \geqslant x_{1}$ до $y>x$, выводим неравенство

$$
-u^{\prime}(x)^{2} \leqslant \beta^{2}\left(u^{2}(y)-u^{2}(x)\right)
$$

После перехода в нем к пределу при $y \rightarrow \infty$ возникает неравенство (1.4), из которого, как и выше, получаем оценку (1.3).

ЛЕмма 1.2. На компактном промежутке $I=(s: a \leqslant s \leqslant b)$ рассмотрим задачу

$$
\begin{gathered}
v^{\prime \prime}(s)=g(s, v), \\
v(a)=A, \quad v(b)=B,
\end{gathered}
$$

где $A, B$ конечны. Пусть найдутся функиии $\alpha(s), \beta(s)$ такие, что

1) $\alpha(s) \leqslant \beta(s) \quad \forall s \in I$

2) $\alpha^{\prime \prime}(s) \geqslant g(s, \alpha(s)), \quad \beta^{\prime \prime}(s) \leqslant g(s, \beta(s)) \quad \forall s \in I$;

3) $\alpha(a) \leqslant A \leqslant \beta(a), \quad \alpha(b) \leqslant B \leqslant \beta(b)$.

Тогда существует решение задачи (1.5), (1.6), которое удовлетворяет неравенству

$$
\alpha(s) \leqslant v(s) \leqslant \beta(s) .
$$

Доказательство приведено, например, в [17; гл. $3, \S 1]$.

\section{$\S 2$. Асимптотика при $x \rightarrow \infty$ для ограниченных значений $t$}

Исходя из изложенных во введении наводящих соображений потребуем, чтобы искомое решение $u(x, t)$ ОДУ (0.1) было нечетной монотонно растушей функцией, которая при больших значениях $x$ близка к $H(x, t)$. Под $H(x, t)$ будем понимать такой корень кубического уравнения $(0.11)$, чтобы функция $H(x, t)$ являлась гладкой всюду в $\mathbb{R}^{2}$, кроме луча $(x=0, t \geqslant 0)$, где $H( \pm 0, t)= \pm \sqrt{t}$. Очевидно, что эта функция $H(x, t)$ монотонно растет и нечетна. (Из ее нечетности следует, в частности, что $H(0, t)=0$ при $t<0$.)

Наложив дополнительные условия

$$
\begin{gathered}
u(0, t)=0, \\
\lim _{x \rightarrow \infty}(u(x, t)-H(x, t))=0,
\end{gathered}
$$

построим решение $u(x, t)$ при $x \geqslant 0$ и продолжим его по нечетности. При этом будем считать, что $H(0, t)=\sqrt{t}$ при $t>0$.

Сначала будем доказывать сушествование $u(x, t)$ без исследования его асимптотики при $x \rightarrow \infty$. Для этого рассмотрим решение $u_{\alpha}(x, t)$ начальной задачи для уравнения $(0.1)$ с условиями $(2.1)$ и

$$
u_{x}^{\prime}(0, t)=\alpha .
$$

Выберем $\alpha=\alpha_{0}$ так, чтобы решение $u_{\alpha_{0}}(x, t)$ задачи Коши $(0.1),(2.1),(2.3)$ было искомым монотонным решением задачи $(0.1),(2.1),(2.2)$. 
При каждом фиксированном $t$ пусть $M(t)$ - множество тех $\alpha$, при которых сушествует точка $x(\alpha, t)>0$ такая, что

$$
0<u_{\alpha}(x, t)<H(x, t)
$$

при $0<x<x(\alpha, t)$ и

$$
u_{\alpha}(x(\alpha, t), t)=H(x(\alpha, t), t) .
$$

Прежде всего отметим, что всюду, кроме начала координат, справедливо неравенство

$$
H_{x}^{\prime}=\frac{1}{3 H^{2}-t}>0
$$

влекушее за собой вывод о том, что $H(x, t) \geqslant H(0, t)$. Последнее неравенство при $t>0$ означает справедливость оценки $H(x, t) \geqslant \sqrt{t}$. Поэтому из соотношения

$$
u^{3}-t u-x=(u-H(x, t))\left(u^{2}+u H(x, t)+H^{2}(x, t)-t\right)
$$

следует, что

$$
u^{3}-t u-x<0
$$

при $0<u<H(x, t)$.

ЛЕмма 2.1. Для любого $t$ множество $M(t)$ непусто.

ДокАЗАТЕльСТво. Вторая производная корня $H(x, t)$ по $x$

$$
H_{x x}^{\prime \prime}=-\frac{6 H}{\left(3 H^{2}-t\right)^{3}}
$$

неположительна всюду, кроме начала координат. Поэтому при $t \neq 0$ из утверждения леммы 1.2 следует, что на отрезке

$$
0 \leqslant x \leqslant 1
$$

существует функция $\widehat{u}(x, t)$, которая удовлетворяет ОДУ $(0.1)$, начальному условию (2.1), краевому условию

$$
u(1, t)=H(1, t)
$$

и, кроме того, двойному неравенству

$$
0 \leqslant \widehat{u}(x, t) \leqslant H(x, t)
$$

(в формулировке леммы 1.2 переменной $s$ соответствует переменная $x$, промежутку $I$ - отрезок $[0,1]$, решению краевой задачи $(1.5),(1.6)$ - решение $\widehat{u}(x, t)$, нижнему барьеру $\alpha(s)$ - тождественный нуль, а верхнему барьеру $\beta(s)$ - функция $H(x, t)$ ). Так как $\widehat{u}(x, t)$ не совпадает с $H(x, t)$, то при $t \neq 0$ в качестве $\alpha \in M(t)$ можно взять $(\widehat{u})_{x}^{\prime}(0, t)$.

Из леммы 1.2 следует также, что при $t=0$ на промежутке (2.6) определено решение $\widetilde{u}(x)$ краевой задачи $(0.1),(2.1),(2.7)$, которое удовлетворяет двойному неравенству $0 \leqslant \widetilde{u}(x) \leqslant 1$. Поскольку

$$
\widetilde{u}(x)<H(x, 0)=x^{1 / 3}
$$

при всех достаточно малых положительных $x$ и $H(1,0)=1$, то это двойное неравенство позволяет утверждать, что $\alpha=(\widetilde{u})^{\prime}(0) \in M(0)$. Лемма доказана. 
ЛЕмма 2.2. Для $\alpha \in M(t)$ при $0 \leqslant x \leqslant x(\alpha, t)$ справедливо неравенство

$$
\frac{\partial u_{\alpha}(x, t)}{\partial x}>0 \text {. }
$$

ДоКАЗАТЕЛЬСТВО. Из принадлежности $\alpha$ к $M(t)$ следует положительность $\alpha$. В самом деле, неравенство $\alpha<0$ невозможно по определению. Предположение же о том, что $\alpha=0$, означает справедливость в точке $x=0$ равенств

$$
u_{x}^{\prime}=u_{x x}^{\prime \prime}=0, \quad u_{x x x}^{\prime \prime \prime}=-1
$$

чего тоже не может быть для положительного при $x>0$ решения $u(x, t)$.

Предположение об обращении $u_{\alpha}(x, t)_{x}^{\prime}$ в нуль в точке $x(\alpha, t)$ противоречит тому, что $H_{x}^{\prime}(x(\alpha, t), t)>0$.

В силу неравенства $(2.5)$ внутри промежутка $[0, x(\alpha, t)]$

$$
\frac{\partial^{2} u_{\alpha}(x, t)}{\partial x^{2}}<0
$$

Из этого неравенства и условий $(2.1),(2.4)$ следует положительность $u_{\alpha}(x, t)_{x}^{\prime}$ и при $0<x<x(\alpha, t)$. Лемма доказана.

ЛЕмма 2.3. При каждом фиксированном $t$ множсество $M(t)$ открьтоо.

ДокАЗАТЕЛЬСтво. Если $\alpha \in M(t)$, то $u_{\alpha}(x, t)<H(x, t)$ при $0<x<x(\alpha, t)$,

$$
u_{\alpha}(x(\alpha, t), t)=H(x(\alpha, t), t)
$$

Следовательно,

$$
\frac{\partial u_{\alpha}}{\partial x} \geqslant \frac{\partial H}{\partial x}
$$

в точке $x(\alpha, t)$. Так как

$$
\frac{\partial^{2} u_{\alpha}}{\partial x^{2}}(x(\alpha, t), t)=0, \quad \frac{\partial^{2} H}{\partial x^{2}}(x(\alpha, t), t)<0,
$$

то $u_{\alpha}>H$ при $x>x(\alpha, t)$ в малой окрестности точки $(x(\alpha, t), t)$ и при малом возмушении $\alpha$ это состояние сохранится. Лемма доказана.

Для каждого $t$ положим

$$
\alpha_{0}(t)=\inf _{\alpha(t) \in M(t)} \alpha(t)
$$

и определим искомую функцию как $u_{\alpha_{0}(t)}(x, t)$. Всюду в дальнейшем под $u(x, t)$ будем понимать именно данное решение. Очевидно, что для любых $x, t$ имеет место неравенство

$$
0 \leqslant u(x, t) \leqslant H(x, t)
$$

и что $u(x, t)$ есть монотонно растущая функция, которая удовлетворяет уравнению (0.1). Из самого способа его построения кажется почти очевиднњм, что оно также удовлетворяет условию (2.2) - т.е. является искомым. Осталось лишш исследовать асимптотику $u(x, t)$ на бесконечности. 
Начнем с анализа асимптотики этой функции при $x \rightarrow \infty$ для конечных значений параметра $t$ (результатом этого анализа, в частности, будет строгое обоснование справедливости для функции $u(x, t)$ соотношения $(2.2))$. Для этого заметим прежде всего, что существует однозначно определенное формальное решение уравнения (0.1) в виде ряда

$$
u=x^{1 / 3}\left(1+\sum_{j=1}^{\infty} c_{j}(t) x^{-2 j / 3}\right) .
$$

Подстановка этого ряда в уравнение (0.1) и приравнивание в результате членов при одинаковых степенях $x$ позволяют последовательно определить все коэффициенты $c_{j}(t)$. Оказывается, что на любом компактном промежутке изменения параметра $t$ при $x \rightarrow \infty$ функция $u(x, t)$ имеет асимптотику в виде ряда $(2.9)$.

ТЕОРема 2.1. Для любого конечного положительного $T_{*}$ существуют такие положительные постоянные $x\left(T_{*}\right)$, что для любого натурального $n$ найдутся полохительные постоянные $M_{n}\left(T_{*}\right)$ и $Q_{n}\left(T_{*}\right)$, для которых в полуполосе

$$
\Lambda=\left(x, t: x>x\left(T_{*}\right), 0 \leqslant|t| \leqslant 2 T_{*}\right)
$$

функиия $и(x, t)$ икоэффициенты $c_{j}(t)$ формального решения ОДУ $(0.1)$ в виде правой части (2.9) связаны неравенствами

$$
\begin{gathered}
\left|u(x, t)-x^{1 / 3}\left(1+\sum_{j=1}^{n-1} c_{j}(t) x^{-2 j / 3}\right)\right| \leqslant M_{n}\left(T_{*}\right) x^{(1-2 n) / 3} \\
\left|u_{x}^{\prime}(x, t)-x^{-2 / 3}\left(\frac{1}{3}+\sum_{j=1}^{n-1} \frac{(1-2 j) c_{j}(t)}{3} x^{-2 j / 3}\right)\right| \leqslant Q_{n}\left(T_{*}\right) x^{-2(1+n) / 3} .
\end{gathered}
$$

ДоказАТЕЛЬСтво. Нетрудно видеть, что коэффициенты $c_{j}(t)$ суть многочлены. Поэтому для любого натурального $n$ и достаточно больших $x\left(T_{*}\right)$ существует постоянная $M_{n}\left(T_{*}\right)$ такая, что в полуполосе (2.10) функции

$$
\begin{aligned}
& Y_{-n}(x, t)=x^{1 / 3}\left(1+\sum_{j=1}^{n-1} c_{j}(t) x^{-2 j / 3}-M_{n}\left(T_{*}\right) x^{-2 n / 3}\right) \\
& Y_{+n}(x, t)=x^{1 / 3}\left(1+\sum_{j=1}^{n-1} c_{j}(t) x^{-2 j / 3}+M_{n}\left(T_{*}\right) x^{-2 n / 3}\right)
\end{aligned}
$$

удовлетворяют неравенствам

$$
\begin{gathered}
\left(Y_{-n}(x, t)\right)_{x x}^{\prime \prime}-\left(Y_{-n}(x, t)\right)^{3}+t Y_{-n}(x, t)+x>0 \\
\left(Y_{+n}(x, t)\right)_{x x}^{\prime \prime}-\left(Y_{+n}(x, t)\right)^{3}+t Y_{+n}(x, t)+x<0 .
\end{gathered}
$$

Следовательно, для достаточно больших значений $x\left(T_{*}\right)$ разности

$$
R_{-}(x, t)=Y_{-n}(x, t)-u(x, t), \quad R_{+}=u(x, t)-Y_{+n}(x, t)
$$


в полуполосе (2.10) удовлетворяют неравенствам

$$
\left(R_{\mp}(x, t)\right)_{x x}^{\prime \prime}>Q_{\mp}(x, t) R_{\mp}(x, t)
$$

с коэффициентами

$$
Q_{\mp}(x, t)=u^{2}(x, t)+u(x, t) Y_{\mp}(x, t)+\left(Y_{\mp}(x, t)\right)^{2}-t>1 .
$$

Согласно лемме 1.1 из этих неравенств и неравенства (2.8) следует, что в полуполосе $(2.10)$ для функций $R_{\mp}(x, t)$ справедливы оценки

$$
R_{\mp}(x, t) \leqslant \mid R_{\mp}\left(x\left(T_{*}\right) \mid \exp \left(x\left(T_{*}\right)-x\right) .\right.
$$

С другой стороны, при $x\left(T_{*}\right) \rightarrow \infty$ имеют место неравенства

$$
0 \leqslant u\left(x\left(T_{*}\right), t\right) \leqslant H\left(x\left(T_{*}\right), T\right)<2\left(x\left(T_{*}\right)\right)^{1 / 3}
$$

и

$$
\left|x\left(T_{*}\right)^{1 / 3}\left(1+\sum_{j=1}^{n-1} c_{j}\left(T_{*}\right) x\left(T_{*}\right)^{-2 j / 3}\right)\right|<2\left(x\left(T_{*}\right)\right)^{1 / 3} .
$$

Из этих неравенств и оценки (2.13) следует, что, увеличив при необходимости $x\left(T_{*}\right)$ и $M_{n}\left(T_{*}\right)$, мы получим в полуполосе (2.10) справедливость неравенств $(2.11)$.

А из этих оценок вытекает и справедливость оценок (2.12). Действительно, оценки (2.11) гарантируют существование такой последовательности $y_{n}(t)$, что $\lim _{n \rightarrow \infty} y_{n}(t)=\infty$ и $\lim _{n \rightarrow \infty} u_{x}^{\prime}\left(y_{n}(t), t\right)=0$. Поэтому после интегрирования обеих частей уравнения $(0.1)$ на промежутке от $x$ до $y_{n}(t)$ с последующим переходом к пределу при $n \rightarrow \infty$ будем иметь интегральное уравнение

$$
u_{x}^{\prime}(x, t)=-\int_{x}^{\infty}\left(u^{3}(z, t)-t u(z, t)-z\right) d z
$$

Из данного интегрального уравнения, из того факта, что ряд (2.9) есть формальное решение ОДУ $(0.1)$, и из оценок (2.11) искомые оценки (2.12) получаются уже непосредственно. Теорема доказана.

ТЕОРема 2.2. Для всех значений параметра $t$ монотонно возрастающее решение задачи (0.1), (2.1), асимптотическое разложсение которого при $x \rightarrow \pm \infty$ задается рядом (2.9), единственно.

ДокАЗАТЕЛьство. После перехода от функции $u(x, t)$ к обратной функции $x(u, t)$ получаем уравнение

$$
\frac{\partial^{2} x}{\partial u^{2}}=F\left(u, t, x, x^{\prime}\right)
$$

где $F\left(u, t, x, x^{\prime}\right)=\left(x+u t-u^{3}\right)(\partial x / \partial u)^{3}$.

Допустим сушествование двух различных решений $u_{1}(x, t)$ и $u_{2}(x, t)$, рассматриваемых в теореме 2.1. Тогда соответствуюшие решения $x_{1}(u, t)$ и $x_{2}(u, t)$ монотонно растут и равны нулю при $u=0$. 
По доказанному выше они имеют одинаковые асимптотические разложения при $u \rightarrow \infty$

$$
x=u^{3}\left(1+\sum_{k=1}^{\infty} d_{k}(t) u^{-k}\right) .
$$

Значит, разность двух таких решений стремится к нулю при $u \rightarrow \infty$.

Эта разность не может иметь ни точек положительного максимума, ни точек отрицательного минимума, так как в этих точках

$$
\frac{\partial F}{\partial x}=\left(\frac{\partial x}{\partial u}\right)^{3}>0
$$

Следовательно, $x_{1}(u, t)=x_{2}(u, t)$. Теорема доказана.

\section{§3. Равномерная асимптотика при $t<0$}

При $t<0$ замена

$$
u=|t|^{1 / 2} w, \quad x=s(-t)^{3 / 2}
$$

сводит решение $u(x, t)$ ОДУ $(0.1)$ к решению $w(s, t)$ ОДУ

$$
t^{-4} w_{s s}^{\prime \prime}=w^{3}+w-s .
$$

Ниже будет построено и обосновано полное асимптотическое разложение при $t \rightarrow-\infty$ этого решения $w(s, t)$, которое будет равномерным по аргументу $s \in \mathbb{R}_{+}$.

Из теоремы 2.1 следует, что нужное нам решение $w(s, t)$ удовлетворяет условиям

$$
\begin{aligned}
w(0, t) & =0, \\
\lim _{s \rightarrow \infty} w_{s}^{\prime}(s, t) & =0, \\
\lim _{s \rightarrow \infty}(w(s, t)-f(s)) & =0,
\end{aligned}
$$

где $f(s)$ есть единственный вещественный корень кубического уравнения

$$
f^{3}+f-s=0 .
$$

Формальное асимптотическое решение (ФАР) задачи (3.1)-(3.4) легко строится в виде ряда

$$
w_{-}(s, t)=\sum_{k=0}^{\infty} t^{-4 k} w_{k}(s),
$$

главный член которого

$$
w_{0}(s)=f(s)
$$

есть корень кубического уравнения (3.5), а остальные выражаются через него из рекуррентной последовательности уравнений, возникающей после подстановки ряда (3.6) в ОДУ (3.1) и приравнивания в результате членов при одинаковых степенях $t$ :

$$
\begin{aligned}
& w_{1}(s)\left(3 f^{2}(s)+1\right)=\left(w_{0}\right)_{s s}^{\prime \prime}, \\
& w_{k}(s)\left(3 f^{2}(s)+1\right)=\left(w_{k-1}\right)_{s s}^{\prime \prime}-\sum_{i_{1}+i_{2}+i_{3}=k, 0 \leqslant i_{j}<k} w_{i_{1}} w_{i_{2}} w_{i_{3}}, \quad k \geqslant 2 .
\end{aligned}
$$


ЛЕмма 3.1. Функиии $w_{k}(s)$, определяемые соотношениями (3.7), (3.8), удовлетворяют условиям

$$
w_{k}(0)=0
$$

и при всех достаточно больших значениях $s>s_{*}$ представляются сходящимися рядами

$$
w_{k}(s)=s^{(1-8 k) / 3} \sum_{j=0}^{\infty} c_{j k} s^{-2 j / 3}
$$

ДокАЗАТЕльство. Для функции $w_{0}(s)$ утверждение леммы следует из кубического уравнения (3.5), а для остальных тривиальным образом доказывается с помощью использования рекуррентных уравнений (3.8) и математической индукции.

Лемма 3.2. Для всех функиий $w_{k}(s)$ существуют положительные постоянные $C_{k}$ такие, что при $s \geqslant 0$ имеют место оценки

$$
\left|w_{k}(s)\right| \leqslant C_{k} \frac{s}{\left(1+s^{2}\right)^{(4 k+1) / 3}} .
$$

ДоКАЗАТЕЛЬСТвО. Из справедливости разложения (3.10) и условия (3.9) следует сушествование таких положительных констант $\alpha_{k}, \beta_{k}$ и $s_{k}$, что при $s \geqslant s_{*}$ и $0 \leqslant s \leqslant s_{k}$ соответственно справедливы оценки $(3.11)$, в которых постоянные $C_{k}$ заменены на константы $\alpha_{k}$ и на константы $\beta_{k}$. Сушествование положительной константы $\gamma_{k}$, обеспечивающей после ее подстановки вместо $C_{k}$ справедливость подобной оценки на компактном интервале $s_{k} \leqslant s \leqslant s_{*}$, очевидно в силу гладкости обеих частей неравенства (3.11). Полагая теперь $C_{k}=\max \left\{\alpha_{k}, \beta_{k}, \gamma_{k}\right\}$, приходим к выводу о справедливости утверждения леммы.

Введем в рассмотрение функции

$$
\begin{aligned}
& Y_{n}(s, t)=\sum_{k=0}^{n-1} \frac{w_{k}(s)}{t^{4 k}}-\frac{K_{n}}{t^{4 n}} \frac{s}{\left(1+s^{2}\right)^{(4 n+1) / 3}}, \\
& Z_{n}(s, t)=\sum_{k=0}^{n-1} \frac{w_{k}(s)}{t^{4 k}}+\frac{K_{n}}{t^{4 n}} \frac{s}{\left(1+s^{2}\right)^{(4 n+1) / 3}} .
\end{aligned}
$$

ЛЕмма 3.3. Функиии $Y_{n}(s, t)$ и $Z_{n}(s, t)$ удовлетворяют условиям

$$
\begin{aligned}
Y_{n}(0, t) & =Z_{n}(0, t)=0, \\
\lim _{s \rightarrow \infty}\left(Y_{n}(s, t)-f(s)\right) & =\lim _{s \rightarrow \infty}\left(Z_{n}(s, t)-f(s)\right)=0 .
\end{aligned}
$$

Для достаточно больиих значений $|t|$ и постоянных $K_{n}$ nри всех $s \geqslant 0 u$ $n>0$ они удовлетворяют неравенствам

$$
\begin{aligned}
& t^{-4} \frac{\partial^{2} Y_{n}}{\partial s^{2}}-Y_{n}^{3}-Y_{n}+s \geqslant 0 \\
& t^{-4} \frac{\partial^{2} Z_{n}}{\partial s^{2}}-Z_{n}^{3}-Z_{n}+s \leqslant 0 .
\end{aligned}
$$


ДоКАЗАТЕЛЬСтво. Справедливость равенств (3.12) и (3.13) очевидным образом следует из соотношений (3.9), (3.10).

Из процесса построения рекуррентной последовательности функций $w_{k}(s)$ следует, что левая часть (3.14) представляет собой многочлен по степеням $t^{-4 j}$

$$
\sum_{j=n}^{3 n} \frac{y_{j}(s)}{t^{4 j}},
$$

для всех коэффициентов $y_{j}(s)$ которого сушествуют положительные постоянные $M_{j}$ такие, что при $s \geqslant 0$ справедливы оценки

$$
\left|y_{j}(s)\right| \leqslant M_{j} \frac{s}{\left(1+s^{2}\right)^{4 j / 3}} .
$$

(Их справедливость следует из оценок (3.11).)

При этом главный член суммы (3.16) при $s>0$ положителен для достаточно больших значений постоянной $K_{n}$, так как

$$
y_{n}(s)=K_{n} \frac{s\left(3 f^{2}+1\right)}{\left(1+s^{2}\right)^{(4 n+1) / 3}}+g_{n}(s),
$$

где функции $g_{n}(s)$, явным образом выражаясь через $s$ и функции $w_{k}(s)$ с номерами $k<n$, удовлетворяют оценкам

$$
g_{n}(s) \leqslant M(n) \frac{s}{\left(1+s^{2}\right)^{4 n / 3}}
$$

с не зависящей от $K_{n}$ постоянной $M(n)$. Из этого факта и из оценок (3.17) для достаточно больших значений $|t|$ сразу же следует справедливость неравенств (3.14). Доказательство неравенства (3.15) совершенно аналогично.

ЛЕмма 3.4. Для решения ОДУ (3.1), удовлетворяющего (3.2)-(3.4), при всех $s \geqslant 0, n>0$ и достаточно больших $|t|$ справедливы неравенства

$$
Y_{n}(s, t) \leqslant w(s, t) \leqslant Z_{n}(s, t) .
$$

ДоКАЗАТЕЛЬСТво. Из вида уравнения (3.1) и из неравенства (3.15) следует, что при $s>0$ разность $R=Z_{n}-w$ удовлетворяет неравенству

$$
t^{-4} R_{s s}^{\prime \prime}<R Q(s, t)
$$

где

$$
Q(s, t)=Z_{n}^{2}+Z_{n} w+w^{2}+1>1 .
$$

Кроме того, соотношения $(3.2),(3.4),(3.7),(3.9)$ и (3.10) означают, что для нее вьполнены условия

$$
R(0)=0, \quad R(\infty)=0
$$

Так как неравенства (3.19), (3.20) препятствуют наличию у функции $R$ отрицательного минимума, то из этих условий следует справедливость второй части неравенства (3.18). Так же доказьвается и его первая часть. 
ТЕОРема 3.1. При $n>1$ и достаточно большом значении $T_{*}>0$ решение задачи (3.1)-(3.4) в квадранте

$$
s \geqslant 0, \quad t \leqslant-T_{*}
$$

допускает оценки

$$
\begin{aligned}
w(s, t)-\sum_{k=0}^{n-1} t^{-4 k} w_{k}(s) & =O\left(t^{-4 n} \frac{s}{\left(1+s^{2}\right)^{(4 n+1) / 3}}\right), \\
w_{s}^{\prime}(s, t)-\sum_{k=0}^{n-2} t^{-4 k} w_{k}^{\prime}(s) & =O\left(t^{-4(n-1)} \frac{1}{\left(1+s^{2}\right)^{4 n / 3-1}}\right) .
\end{aligned}
$$

ДоКАЗАТЕЛЬСТВо. Из оценок (3.11) следует, что соотношения (3.21) доказаны в лемме 3.4. А уже из этих соотношений, (3.3), вида ОДУ (3.1), рекуррентной последовательности формул (3.7), (3.8) и очевидных оценок

$$
w_{k}^{\prime}(s)=O\left(\frac{1}{\left(1+s^{2}\right)^{(4 k+1) / 3}}\right)
$$

вьводится справедливость оценок (3.22).

В самом деле,

$$
\begin{aligned}
w_{s}^{\prime}(s, t) & =-t^{4} \int_{s}^{\infty}\left(w^{3}(y, t)-w(y, t)-y\right) d y \\
& =-\int_{s}^{\infty}\left(\sum_{j=0}^{n-2} t^{-4 j}\left(w_{j}(y, t)\right)_{y y}^{\prime \prime}+O\left(t^{-4(n-1)} \frac{y}{\left(1+y^{2}\right)^{4 n / 3}}\right)\right) d y .
\end{aligned}
$$

Поэтому

$$
w_{s}^{\prime}(s, t)-\sum_{j=0}^{n-2} t^{-4 j}\left(w_{j}(s, t)\right)_{s}^{\prime}=O\left(t^{-4(n-1)} \frac{1}{\left(1+s^{2}\right)^{4 n / 3-1}}\right) .
$$

Теорема доказана.

Из этой теоремы следует, что формальное решение $w_{-}(s, t)$ действительно является равномерньм по $s$ асимптотическим разложением интересующего нас решения задачи (3.1)-(3.4).

\section{§4. Равномерная асимптотика при $t>0$}

При $t>0$ замена $(0.12)$ переводит функцию $u(x, t)$ в гладкое и монотонное решение уравнения $(0.14)$, которое удовлетворяет условиям

$$
\begin{gathered}
v(0, t)=0, \\
\lim _{s \rightarrow \infty} v_{s}^{\prime}(s, t)=0, \\
\lim _{s \rightarrow \infty}(v(s, t)-f(s))=0,
\end{gathered}
$$

где $f(s)$ есть гладкий и положительньй при $s \geqslant 0$ корень кубического уравнения (0.13). Для $v(s)$ справедливо двойное неравенство

$$
0 \leqslant v(s, t) \leqslant f(s) .
$$


$\Phi$ АР уравнения (0.14), формально удовлетворяющее условиям (4.2), (4.3), нетрудно построить в виде ряда

$$
v_{+}(s, t)=\sum_{k=0}^{\infty} t^{-4 k} v_{k}(s)
$$

главный член которого

$$
v_{0}(s)=f(s)
$$

есть упомянутый положительный корень кубического уравнения (0.13), а остальные выражаются через него из рекуррентной последовательности уравнений, возникающей в результате подстановки ряда (4.5) в ОДУ (0.14) и приравнивания членов при одинаковых степенях $t$ :

$$
\begin{aligned}
& v_{1}(s)\left(3 f^{2}(s)-1\right)=\left(v_{0}\right)_{s s}^{\prime \prime}, \\
& v_{k}(s)\left(3 f^{2}(s)-1\right)=\left(v_{k-1}\right)_{s s}^{\prime \prime}-\sum_{i_{1}+i_{2}+i_{3}=k, 0 \leqslant i_{j}<k} v_{i_{1}} v_{i_{2}} v_{i_{3}}, \quad k \geqslant 2 .
\end{aligned}
$$

Для коэффициентов этого ФАР справедливы следующие три леммы, доказательство которых фактически сводится к повторению рассуждений, использованных при доказательстве лемм 3.1-3.3.

ЛЕмма 4.1. Функиии $v_{k}(s)$, определяемые соотношениями (4.6),(4.7), при всех достаточно больших значениях s представляются сходящимися рядами

$$
v_{k}(s)=s^{(1-8 k) / 3} \sum_{j=0}^{\infty} c_{j k} s^{-2 j / 3}
$$

Лемма 4.2. Существуют положительнье постоянные $C_{k}$ такие, что при $s \gg 1$ имеют место оценки

$$
\left|v_{k}(s)\right| \leqslant C_{k} s^{(1-8 k) / 3} .
$$

ЛЕмма 4.3. Для любых постоянных $K_{n}$ функиии

$$
A_{n}(s, t)=\sum_{k=0}^{n-1} \frac{v_{k}(s)}{t^{4 k}}-\frac{K_{n}}{t^{4 n}} s^{(1-8 n) / 3}, \quad B_{n}(s, t)=\sum_{k=0}^{n-1} \frac{v_{k}(s)}{t^{4 k}}+\frac{K_{n}}{t^{4 n}} s^{(1-8 n) / 3}
$$

удовлетворяют условиям

$$
\lim _{s \rightarrow \infty}\left(A_{n}(s, t)-f(s)\right)=0, \quad \lim _{s \rightarrow \infty}\left(B_{n}(s, t)-f(s)\right)=0 .
$$

Для достаточно больиих значений постоянных $s_{*}, K_{n}, T$ и при всех $s \geqslant s_{*}$ $u t \geqslant T$ имеют место неравенства

$$
t^{-4} \frac{\partial^{2} A_{n}}{\partial s^{2}}-A_{n}^{3}+A_{n}+s \geqslant 0, \quad t^{-4} \frac{\partial^{2} B_{n}}{\partial s^{2}}-B_{n}^{3}+B_{n}+s \leqslant 0 .
$$


ЛЕмма 4.4. Пусть функиия $v(s, t)$ удовлетворяет условиям (4.2), (4.3) и является решением ОДУ (0.14) при всех $s$, бо́льших некоторой положительной постоянной. Тогда для достаточно больиих значений $t$ и постоянных $s^{*}, K_{n-1}$ оно при $n \geqslant 1 u s \geqslant s^{*}$ удовлетворяет двойному неравенству

$$
A_{n-1}(s, t) \leqslant v(s, t) \leqslant B_{n-1}(s, t) .
$$

ДоказАТЕЛЬСтво. Докажем левую часть неравенства (4.12). Из вида уравнения (0.14) и первого из неравенств (4.11) следует, что при достаточно больших значениях $K_{n}$ разность $R=A_{n}-v$ удовлетворяет неравенству

$$
t^{-4} R_{s s}^{\prime \prime}>R Q(s, t)
$$

где в силу справедливости соотношений (4.8) функция

$$
Q(s, t)=A_{n}^{2}+A_{n} v+v^{2}-1
$$

при $s$, бо́льших некоторой константы $s^{*}>0$, допускает оценку

$$
Q(s, t)>1 \text {. }
$$

Кроме того, соотношения (4.2), (4.3) и (4.10) означают, что для $R$ выполнены условия

$$
R(\infty)=R^{\prime}(\infty)=0 .
$$

Согласно лемме 1.1 из соотношений (4.13)-(4.15) следует неравенство $|R(s, t)| \leqslant$ $\left|R\left(s^{*}, t\right)\right| \exp \left(t^{2}\left(s^{*}-s\right)\right)$. Учитывая неравенство (4.4), приходим к выводу о справедливости левой части неравенства (4.12) для достаточно больших значений постоянных $K_{n-1}$.

Доказательство правой части неравенства (4.12) проводится аналогично вышеприведенному.

Повторение рассуждений, использованных при доказательстве теоремы 3.1, приводит теперь к заключению об асимптотическом характере формального решения в виде ряда (4.5).

Теорема 4.1. При достаточно больиих значениях постоянных $T_{*}$ и $S_{*}$ для решения $v(s, t)$ ОДУ (0.14), удовлетворяющего условиям (4.1)-(4.3), в квадранте

$$
\Upsilon: \quad s \geqslant S_{*}, \quad t>T_{*}
$$

справедливы оценки

$$
v(s, t)-\sum_{k=0}^{n-1} t^{-4 k} v_{k}(s)=O\left(t^{-4 n} s^{(1-8 n) / 3}\right),
$$

здесь функции $v_{k}(s)$, определяемые формулами (4.6), (4.7), удовлетворяют оценкам (4.9).

Таким образом, осталось исследовать асимптотику при $t \rightarrow \infty$ на конечном промежутке

$$
J: 0 \leqslant s \leqslant S_{*}
$$


монотонного решения ОДУ $(0.14)$, которое удовлетворяет начальному условию (4.1) и краевому условию

$$
v\left(S_{*}, t\right)=v_{*}(t)
$$

Здесь функция $v_{*}(t)$ при $t \rightarrow \infty$ имеет АР

$$
v_{*}(t)=\sum_{k=0}^{\infty} t^{-4 k} v_{k}\left(S_{*}\right)
$$

где $v_{k}(s)$ заданы формулами (4.6), (4.7).

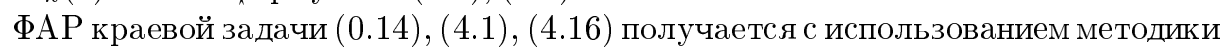
экспоненциально убывающих пограничных функций [18]. Оно имеет вид суммы

$$
v=\sum_{j=0}^{\infty} t^{-4 j} v_{j}(s)+\sum_{k=0}^{\infty} t^{-2 k} \Pi_{k}(\xi)=v_{+}(s, t)+\Pi(\xi, t)
$$

формального решения $v_{+}(s, t)$ ОДУ $(0.14)$ в виде ряда $(4.5)$ (как следует из рекуррентных соотношений $(4.6),(4.7)$, его коэффициенты $v_{k}(s)$ являются гладкими при $s \geqslant 0$ функциями) и зависящего от растянутой переменной $\xi=s t^{2}$ погранслойного ряда, формально устраняющего невязку, вносимую в начальное условие (4.1) рядом $v_{+}(s, t)$.

Подставляя анзац (4.17) в ОДУ $(0.14)$, получаем, что

$$
t^{-4}\left(v_{+}(s, t)\right)_{s s}^{\prime \prime}+(\Pi(\xi, t))_{\xi \xi}^{\prime \prime}=\left(v_{+}(s, t)\right)^{3}-v_{+}(s, t)-s+\Pi h(\xi, t),
$$

где П $h(\xi, t)$ есть формальный ряд, получающийся в результате подстановки в выражение

$$
\Pi h(\xi, t)=\left(3 v_{+}^{2}\left(\xi t^{-2}, t\right)-1\right) \Pi(\xi, t)+3 v_{+}\left(\xi t^{-2}, t\right)(\Pi(\xi, t))^{2}+(\Pi(\xi, t))^{3}
$$

рядов $\Pi(\xi, t)$ и $v_{+}\left(\xi t^{-2}, t\right)$, в последнем из которых все коэффициенты $v_{k}\left(\xi t^{-2}\right)$ заменены их рядами Тейлора в точке $\xi=0$.

Поскольку $v_{+}(s, t)$ есть формальное решение ОДУ (0.12), то подстановка в задачу $(0.14),(4.1)$ анзаца (4.17) и приравнивание в результате подстановки коэффициентов при одинаковых степенях $t$ дает следующую рекуррентную последовательность задач на коэффициенты $\Pi_{k}(\xi)$ погранслойного ряда $\Pi(\xi, t)$ :

$$
\begin{aligned}
& \left(\Pi_{0}\right)_{\xi \xi}^{\prime \prime}=\left(\Pi_{0}\right)^{3}+3\left(\Pi_{0}\right)^{2}+2 \Pi_{0}, \quad \Pi_{0}(0)=-1, \\
& L \Pi_{1} \equiv\left(\Pi_{1}\right)_{\xi \xi}^{\prime \prime}-\left(3\left(\Pi_{0}\right)^{2}+6\left(\Pi_{0}\right)+2\right) \Pi_{1} \\
& =P_{1}\left(\xi, \Pi_{0}\right), \quad \Pi_{1}(0)=0, \\
& L \Pi_{2}=P_{2}\left(\xi, \Pi_{0}, \Pi_{1}\right), \quad \Pi_{2}(0)=-v_{1}(0), \\
& L \Pi_{2 n-1}=P_{2 n-1}\left(\xi, \Pi_{0}, \ldots, \Pi_{2 n-2}\right), \quad \Pi_{2 n-1}(0)=0 \\
& L \Pi_{2 n}=P_{2 n}\left(\xi, \Pi_{0}, \ldots, \Pi_{2 n-1}\right), \quad \Pi_{2 n}(0)=-v_{n}(0) .
\end{aligned}
$$

Здесь $P_{m}$ суть многочлены (индекс $m$ не имеет отношения к степени этих многочленов) своих аргументов, коэффициенты которых зависят лишш от значений в нуле функций $v_{k}(s)$ и значений в нуле их производных с индексом $k \leqslant[m / 2]$. При $\xi=0$ эти многочлены не обращаются тождественно в нуль. 
Задачи (4.18)-(4.22) добавляются стандартньми для метода экспоненциально убываюших погранфункций условиями:

$$
\Pi_{k}(\infty)=0, \quad\left(\Pi_{k}\right)^{\prime}(\infty)=0, \quad k=0,1,2, \ldots .
$$

Решение задачи (4.18), (4.23)

$$
\Pi_{0}(\xi)=-1+\operatorname{th} \frac{\xi}{\sqrt{2}}
$$

есть отрицательная при всех конечных $\xi$ функция с положительной производной

$$
\Phi(\xi)=\left(\Pi_{0}(\xi)\right)_{\xi}^{\prime}=\frac{1}{\sqrt{2}(\operatorname{ch}(\xi / \sqrt{2}))^{2}} .
$$

Лемма 4.5. Существуют положительные постояннье $M_{n}$ и натуральнье числа $j(n)$ такие, что модули решений последовательности задач (4.18)(4.22), удовлетворяющих условиям (4.23), а также модули их первых производных при достаточно больших $\xi$ не превосходят функций

$$
M_{n} \xi^{j(n)} \exp (-\sqrt{2} \xi)
$$

ДокАЗАТЕЛЬСтво. Для $n=0$ утверждение леммы очевидным образом следует из формул (4.24), (4.25). Далее из вида решений задач (4.19)-(4.22)

$$
\Pi_{k}(\xi)=\Phi(\xi)\left(\Pi_{k}(0) \sqrt{2}-\int_{0}^{\xi} \Phi^{-2}(y) \int_{y}^{\infty} \Phi(\sigma) H_{k}(\sigma) d \sigma d y\right),
$$

где $k=1,2, \ldots, H_{k}$ - правые части ОДУ в задачах (4.18)-(4.22), удовлетворяющих условиям (4.23), утверждение леммы следует по индукции [18].

ТеОРема 4.2. Монотонное решение краевой задачи (0.12), (4.1), (4.16) единственно и при $t \rightarrow \infty$ имеет дифференцируемое асимптотическое разложение (4.17).

ДокАЗАТЕЛЬство. Единственность доказывается так же, как в теореме 2.2. Обоснование же асимптотики (4.17) произведем, следуя схеме статьи [19], с помощью леммы 1.2 .

Фигурирующим в формулировке этой леммы постоянным и функциям в нашем случае отвечают следующие:

$$
\begin{gathered}
a=0, \quad b=S_{*}, \quad A=0, \quad B=v\left(S_{*}, t\right), \quad g(s, v)=t^{4}\left(v^{3}-v-s\right), \\
\beta(s) \equiv \beta_{4 n}(s)=V_{n}(s, t)+t^{-4 n} \gamma_{n}\left(V_{n}(s, t)\right)_{s}^{\prime}, \\
\alpha(s) \equiv \alpha_{4 n}(s)=V_{n}(s, t)-t^{-4 n} \gamma_{n}\left(V_{n}(s, t)\right)_{s}^{\prime}
\end{gathered}
$$

где

$$
V_{n}(s, t)=\sum_{k=0}^{n-1} v_{k}(s) t^{-4 k}+\sum_{j=0}^{2 n-1} \Pi_{j}(\xi) t^{-2 j},
$$

а $\gamma_{n}$ - положительная постоянная.

Для достаточно больших значений $\gamma_{n}$ из способа построения функций $\alpha(s)$ и $\beta(s)$ вьполнение условий 1 ) и 3 ) леммы 1.2 следует автоматически (функции $f^{\prime}(s)$ и $\left(\Pi_{0}(\xi)\right)_{\xi}^{\prime}$ положительны на интервале $\left.J\right)$. 
Заметим далее, что имеют место соотношения

$$
\begin{gathered}
t^{-4}\left(V_{n}(s, t)\right)_{s s}^{\prime \prime}-\left(V_{n}(s, t)\right)^{3}+V_{n}(s, t)+s=O\left(t^{-4 n}\right), \\
t^{-4}\left(V_{n}(s, t)\right)_{s s s}^{\prime \prime \prime}-\left(3\left(V_{n}(s, t)\right)^{2}-1\right)\left(V_{n}(s, t)\right)_{s}^{\prime}+1=O\left(t^{-4 n_{1}(n)}\right),
\end{gathered}
$$

где $\lim _{n \rightarrow \infty} n_{1}(n)=\infty$. Поэтому при $t \rightarrow \infty$ для всех достаточно больших значений натурального $n$ найдутся такие постоянные $\gamma_{n}$, что будет выполнено соотношение

$$
\begin{aligned}
& t^{-4} \beta_{s s}^{\prime \prime}(s, t)-\beta^{3}(s, t)+\beta(s, t)+s=t^{-4 n}\left(\gamma_{n}\left(-1+O\left(t^{-4 n_{1}(n)}\right)\right)\right. \\
& \left.\quad+P\left(t, \xi, \Pi_{0}(\xi), \ldots, \Pi_{n-1}(\xi), s, v_{0}(s), \ldots, v_{n-1}(s)\right)\right)
\end{aligned}
$$

где $P$ - ограниченная при $t \rightarrow \infty$ и $0 \leqslant s \leqslant S_{*}$ функция. Поэтому при достаточно больших значениях $n$ положительной постоянной $\gamma_{n}$ правая часть равенства (4.28) отрицательна. Таким образом, при достаточно больших значениях $n$ вьполняется первая часть условий 3$)$ леммы 1.2 , касающаяся верхнего барьера $\beta(s, t)$. Отсюда, очевидно, следует их выполнение и при всех натуральных $n$. Точно так же показывается выполнение второй части этих условий, касающейся нижнего барьера $\alpha(s, t)$. А следовательно, при всех натуральных $n$ барьеры (4.26), (4.27) и решение $v(s, t)$ краевой задачи $(0.12),(4.1),(4.16)$ в силу леммы 1.2 связаны неравенством (1.7), означающим обоснованность разложения (4.17).

Эти неравенства гарантируют также сушествование внутри интервала $J$ точки $s_{i}(t)$, в которой для всех достаточно больших натуральных $n$ имеют место оценки

$$
\left|(\alpha(s, t)-v(s, t))_{s}^{\prime}\right|=O\left(t^{-4 n_{2}(n)}\right), \quad\left|(\beta(s, t)-v(s, t))_{s}^{\prime}\right|=O\left(t^{-4 n_{2}(n)}\right),
$$

где $\lim _{n \rightarrow \infty} n_{2}(n)=0$. Из этого факта, из обоснованности разложения (4.17) и из вида ОДУ (0.14) дифференцируемость (и, как следствие, монотонность соответствуюшего решения $v(s, t))$ теперь вытекает непосредственно. Теорема доказана.

\section{Зак лючение}

Таким образом, получена равномерная асимптотика функции $u(x, t)$ при $x^{2}+t^{2} \rightarrow \infty:$

i) при $|t| \leqslant T_{*}$

$$
\left|u(x, t)-x^{1 / 3}\left(1+\sum_{j=1}^{n-1} c_{j}(t) x^{-2 j / 3}\right)\right| \leqslant M_{n}\left(T_{*}\right) x^{(1-2 n) / 3} ;
$$

ii) при $t<-T^{*}$

$$
\left.\left.|u(x, t)-| t\right|^{1 / 2}\left(\sum_{k=0}^{n-1}|t|^{-4 k} w_{k}(s)\right)\left|\leqslant A_{n}\left(T^{*}\right)\right| t\right|^{1 / 2-4 n} \frac{s}{\left(1+s^{2}\right)^{(4 n+1) / 3}} ;
$$

iii) при $t>T_{* *}$ и $0 \leqslant s \leqslant \infty$

$$
\begin{aligned}
& \left|u(x, t)-t^{1 / 2}\left(\sum_{k=0}^{n-1} v_{k}(s) t^{-4 k}+\sum_{j=0}^{2 n-1} \Pi_{j}(\xi) t^{-2 j}\right)\right| \\
& \leqslant B_{n}\left(T_{* *}\right) t^{1 / 2-4 n}\left((1+s)^{(1-8 n) / 3}+\left|\Pi_{2 n}(\xi)\right|\right) .
\end{aligned}
$$


Здесь $T_{*}, T^{*}, T_{* *}$ - произвольные, а $A_{n}\left(T^{*}\right), B_{n}\left(T^{* *}\right)$ - конкретные положительные постоянные, $s=x /|t|^{3 / 2}, \xi=x t^{1 / 2}$, а $w_{k}(s), v_{k}(s)$ и $\Pi_{k}(\xi)$ - конкретные гладкие функции, определенные в $\S \S 3,4$. Функции $\Pi_{k}(\xi)$ экспоненциально быстро стремятся к нулю при $\xi \rightarrow \infty$.

\section{Список литературы}

1. Ильин А.М., Сулейманов Б.И. О двух специальных функциях, связанњых с особенностью сборки // Докл. РАН. 2002. Т. 387. № 2. С. 156-158.

2. Арнольд В. И. Теория катастроф. М.: Наука, 1980.

3. De Villiers J. M. A uniform asymptotic expansion of the positive solution of a non-linear Dirichlet problem // Proc. London Math. Soc. (3). 1973. V. 27. P. 701-722.

4. Ligthill M. J. Viscosity effects in sound waves of finite amplitude // Surveys in mechanics / ed. G. K. Batchelor, R.M. Davies. Cambridge: Cambridge Univ. Press, 1956. P. 250-351.

5. Ильин A. M. Задача Коши для одного квазилинейного уравнения с мальм параметром // Докл. АН СССР. 1985. Т. 283. № 3. С. 530-534.

6. Haberman R. Note of the initial formation of shocks // SIAM J. Appl. Math. 1986. V. 6. № 1. P. 16-19.

7. Haberman $R$. The initial formation and stucture of two dimensional diffusive shock waves // Wave Motion. 1986. V. 8. P. 267-276.

8. Ильин A. M. Согласование асимптотических разложений решений краевых задач. М.: Наука, 1987.

9. Zakharov S. V., Il'in A.M. On the influence of small dissipation on the evolution of weak discontinuities // Funct. Differ. Equ. 2001. V. 8. № 3-4. P. 257-271.

10. Мизес $P$. Математическая теория течений сжимаемой жидкости. М.: ИЛ, 1961.

11. Богаевский B. H., Повзнер B. Н. Алгебраические методы в нелинейной теории возмущений. М.: Наука, 1987.

12. Маслов В. П., Мосолов П. П. Уравнения одномерного баротропного газа. М.: Наука, 1990 .

13. Фай $Ф$., Гринли У. Внутренние переходные слои для эллиптических краевых задач с мальм параметром // УМН. 1974. Т. 29. №4. С. 103-130.

14. Нефедов Н. Н. Метод дифференциальных неравенств для некоторых классов нелинейных сингулярно возмущенных задач с внутренними слоями // Дифференц. уравнения. 1995. T. 31. № 7. C. 1142-1149.

15. Бутузов В. Ф., Неделько И. В. О неустойчивости многомерных контрастных структур // Дифференц. уравнения. 2002. Т. 38. № 2. С. 222-233.

16. Ильин А.М., Сулейманов Б.И. Коэффициенты внутреннего разложения при исследовании асимптотики некоторых сингулярно возмущенных краевых задач // Дальневост. матем. журн. 2003. Т. 4. № 1. С. 78-85.

17. Васильев Н. И., Клоков Ю. А. Основы теории краевых задач обыкновенных диффференциальных уравнений. Рига: Зинатне, 1978.

18. Васильева А.Б., Бутузов В.Ф. Асимптотические разложения решений сингулярно возмущенных краевых задач обыкновенных дифференциальных уравнений. М.: Наука, 1973.

19. Нефедов H. Н. Метод дифференциальных неравенств для некоторых классов нелинейных сингулярно возмущенных задач в частных производных // Дифференц. уравнения. 1995. T. 31. № 4. C. $719-722$.

Челябинский государственный университет;

Институт математики с ВЦ УфНЦ, Уф̆а

E-mail: iam@csu.go.ru, ilam@go.ru;

Поступила в редакцию

bis@imat.rb.ru

20.05.2004 